
\title{
La intervención social
}

\section{EN UN CENTRO DE EDUCACIÓN ESPECIAL SINGULAR}

http://dx.doi.org/pym.i358.y2014.002

$M^{a}$ Paz de la Fuente Olózaga y Ana Isabel Sanz Jiménez

Profesoras Técnicos de Servicios a la Comunidad. Diplomada en Trabajo Social

cpee.mariasoriano.madrid@educa.madrid.org

cpee.mariasoriano.madrid@educa.madrid.org

n este artículo pretendemos ofrecer una panorámica general sobre la intervención social que realizamos en un colegio de educación especial muy singular, pero para ello es imprescindible hacer previamente una breve reseña del centro y de su longevo pasado, ya que este curso celebramos su centésimo vigésimo quinto aniversario. El centro público de educación especial María Soriano es el heredero directo de una serie de instituciones que siempre han estado respondiendo a necesidades de colectivos afectados de discapacidad: asilo de inválidos del trabajo (1898), instituto de reeducación profesional de inválidos del trabajo (1922), instituto de reeducación profesional (1928), instituto nacional de reeducación de inválidos (1933) y CPEE de reeducación de inválidos (1986).

En la actualidad, existen multitud de elementos que han permanecido a lo largo de su trayectoria y que han hecho de nuestro centro un recurso educativo diferente a los demás, formando parte de las señas de su identidad. Desde el 2006 nos identificamos por CPEE María Soriano. Estamos ubicados en el distrito de Carabanchel de Madrid, aunque nuestro alumnado proviene de municipios diversos de la Comunidad de Madrid, ya que somos de ámbito regional.

La población escolar a la que nos dirigimos presenta discapacidad motora, con otras asociadas como la intelectual fundamentalmente, y cuyas necesidades educativas especiales (NEE) no puedan ser atendidas dentro de los centros ordinarios. Nuestro alumnado tiene edades comprendidas entre los 3 y los 21 años, muchos de ellos requieren de atenciones sanitarias y ayuda para todas las actividades de la vida diaria. El número de alumnos matriculados en estos momentos es 8I, la mayoría están escolarizados en régimen de externado, sólo contamos con plazas de residencia para un máximo de 30 alumnos. Las familias de los alumnos residentes solicitan este tipo de plaza debido a circunstancias socio familiares y/o sanitarias determinadas. También cuenta con un programa "Respiro" destinado a todos los alumnos escolarizados en el centro, es un recurso residencial con carácter temporal, consideramos conveniente

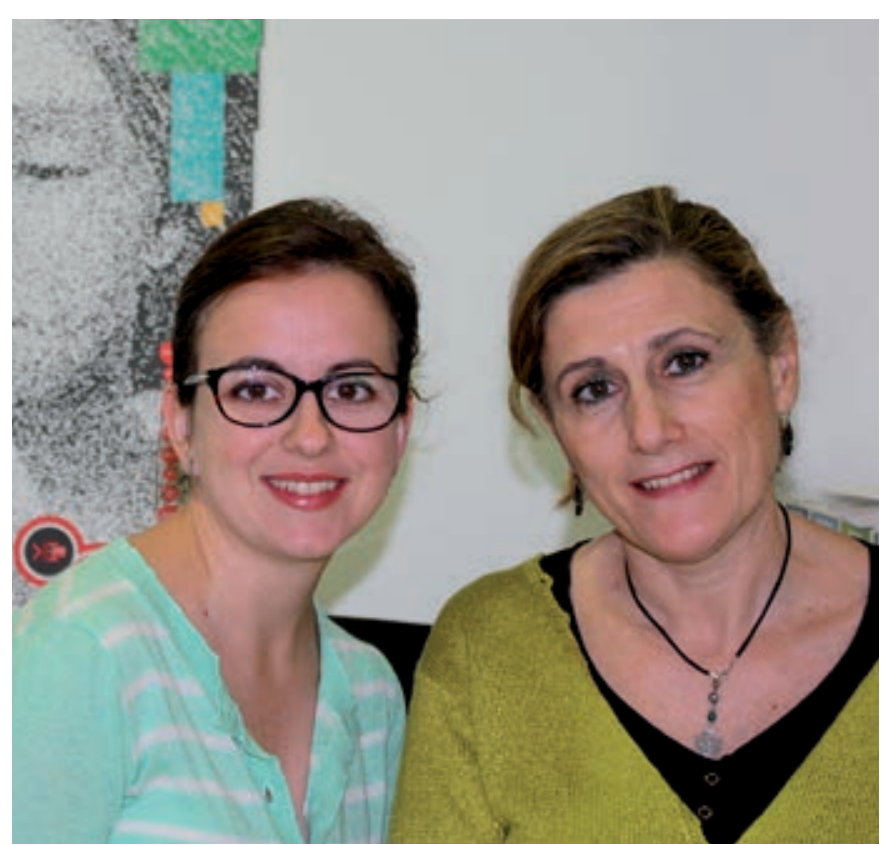

Ma Paz de la Fuente Olózaga y Ana Isabel Sanz Jiménez. 
señalar que la residencia permanece cerrada durante los periodos de vacaciones escolares. A nivel académico hay un total de diez aulas, cuatro de ellas de educación infantil, ocho de EBO y dos de transición a la vida adulta.

La vía de acceso al centro es a través de los equipos educativos de atención temprana (EAT), los equipos de orientación educativa y psicopedagógica (EOEP) y los departamentos de orientación de los centros de origen, son ellos quienes realizan las funciones de valoración y derivación. Posteriormente corresponde al servicio de apoyo a la escolarización específica para alumnado con NEE asociadas a condiciones personales de discapacidad, con graves trastornos de la personalidad y de la conducta, realizar la propuesta final y al servicio de inspección educativa hacerla efectiva.

Una vez que recibimos dicha propuesta de escolarización para un nuevo alumno se pone en funcionamiento el protocolo de valoración de nuevos alumnos, con el objeto de conocer las necesidades del menor y poder ofrecerle una atención adecuada e integral. En esta comisión participan representantes de diferentes ámbitos profesionales del centro que valoran, a través de informes profesionales y entrevistas, las necesidades del alumno y proponen todos aquellos aspectos que se relacionan con su escolarización, aula asignada, servicios/apoyos, aspectos de residencia, etcétera. También se concretan aquellos datos de carácter social que puedan influir o condicionar la evolución favorable del menor y que se deban tener en cuenta para cualquier intervención. Todos los apoyos que se proponen son individuales partiendo de sus capacidades, necesidades específicas y respetando las diferencias culturales o de otra índole.

Otra de las peculiaridades del centro es la variedad de perfiles profesionales que desempeñan su labor en el mismo y que nos diferencian del resto de colegios de educación especial. Muchos de estos perfiles están relacionados con el área sanitaria, donde se cuenta, además de los DUE, con médicos, y médico rehabilitador, auxiliares de clínica y terapeutas ocupacionales. Ésta diversidad de profesionales impone un método de trabajo interdisciplinar donde una coordinación eficaz se vuelve indispensable.

La área de trabajo social, que se encuentra ubicada dentro del departamento de orientación, cuenta con una larga trayectoria en el centro, adaptándose a los cambios que se han ido produciendo, en relación a los alumnos y en la situación laboral de sus componentes, ya que desde 1992 pasaron a formar parte del cuerpo de profesores técnicos de servicios a la comunidad, (PTSC). Actualmente las dos profesionales de este departamento son trabajadoras sociales, pero pueden acceder a este cuerpo un perfil muy variado de titulados.

Estos profesionales de acuerdo con el proyecto educativo del centro colaboran con otros técnicos en el desarrollo integral de los alumnos. Realizan actuaciones en el ámbito escolar, familiar y comunitario, sensibilizando sobre la importancia de las necesidades sociales y su influencia en el proceso educativo. No se trata de una mera gestión e información de recursos sociales, sino que deben ser dinamizadores de los implicados en el proceso educativo y de esta forma prevenir desajustes familiares, conocer el entorno de los alumnos y facilitarles el acceso a los recursos sociales.

Por su trayectoria profesional, en el centro público de educación especial María Soriano, saben cómo la discapacidad genera en muchas de las familias que la viven unas situaciones de exclusión que precisan del acompañamiento profesional y de un apoyo en situaciones de crisis cuando se enfrentan a un problema que en general les desborda.

Las funciones más significativas, con los alumnos, que desarrollan estos profesionales se pueden sintetizar en tres momentos distintos. Todas aquellas relacionadas con la admisión (el primer contacto), con el seguimiento y con la derivación a otro colegio o recurso al finalizar la etapa educativa.

Podemos hacer otra clasificación más completa de las actuaciones que llevan a cabo, dependiendo del ámbito de intervención. En relación al centro, a las familias y a los recursos externos:

- Participan en las distintas reuniones formales que se realizan, aportando propuestas e información, especialmente relacionada con aspectos familiares o de recursos externos (claustro, equipo técnico de residencia, departamento de orientación, comisión de ayudas técnicas, etcétera).

- Coordinan la Comisión de valoración de nuevos alumnos, se trata por una parte de abordar aquellas actuaciones previas a la propuesta de escolarización o solicitud de plaza: como visitas al centro y atención telefónica de familiares y profesionales, y por otra, de poner en funcionamiento el protocolo de esta comisión, coordinando las distintas entrevistas de los profesionales participantes y la posterior

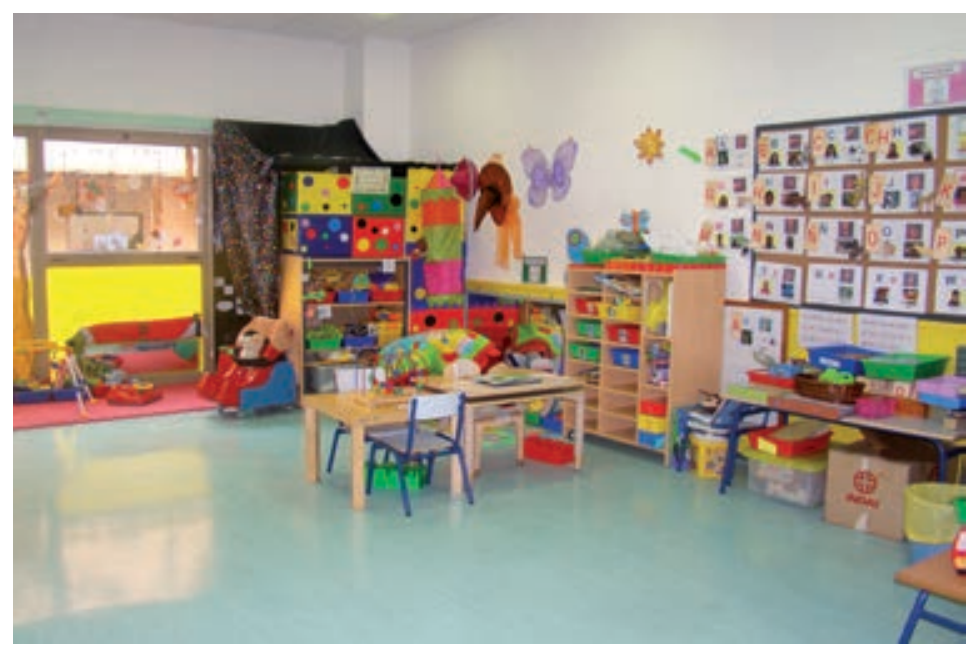


reunión de puesta en común (médicos, enfermera, orientadora, jefa de estudios, jefa de estudios de residencia y PTSC).

- Coordinan con el tutor y el orientador para tratar aspectos relevantes que puedan ser de utilidad para su labor profesional con el alumno.

- Colaboran en la prevención y control del absentismo escolar. Aunque las ausencias del alumnado en este centro suelen estar relacionadas con su salud, en algunos casos son menores con graves enfermedades.

- Sensibilizan e informan a los profesionales del centro de la influencia de las necesidades sociales en la evolución de los alumnos.

- Coordinan el programa "Respiro" existente en el centro. Manteniendo reuniones frecuentes con la jefa de estudios de residencia para abordar aspectos relacionados con los alumnos internos y con solicitudes de programa "Respiro".

- Mantienen una fluida comunicación con los miembros de la Asociación de Madres y Padres (AMPA).

En relación con la familia:

- Con todas se mantiene una entrevista inicial con objeto de informar sobre cuestiones organizativas del centro y también para detectar algún condicionante familiar que pudiera incidir en el proceso educativo del alumno. En bastantes casos son familias con intervención en servicios sociales.

- Dependiendo de la situación, por demanda de estas profesionales o de los padres/responsables, se establece un seguimiento más o menos frecuente. Actualmente se realiza intervención regular con la mayoría de las familias (por la tipología de unidad de convivencia familiar y por la situación económica, entre otras causas).

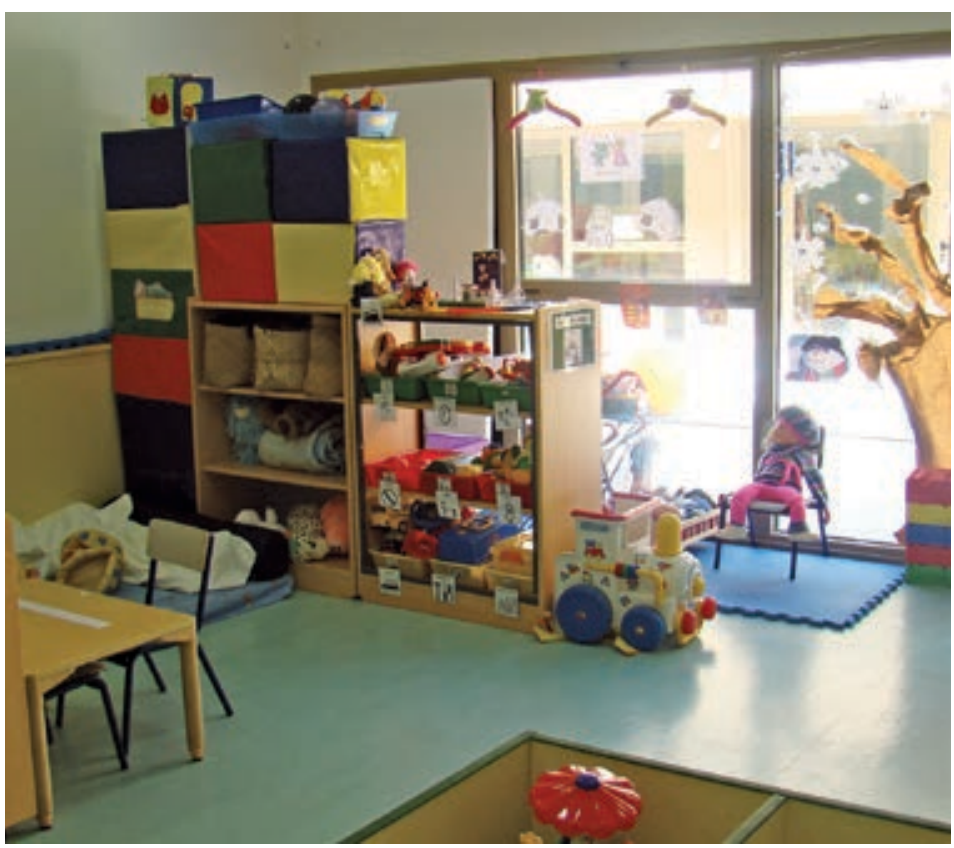

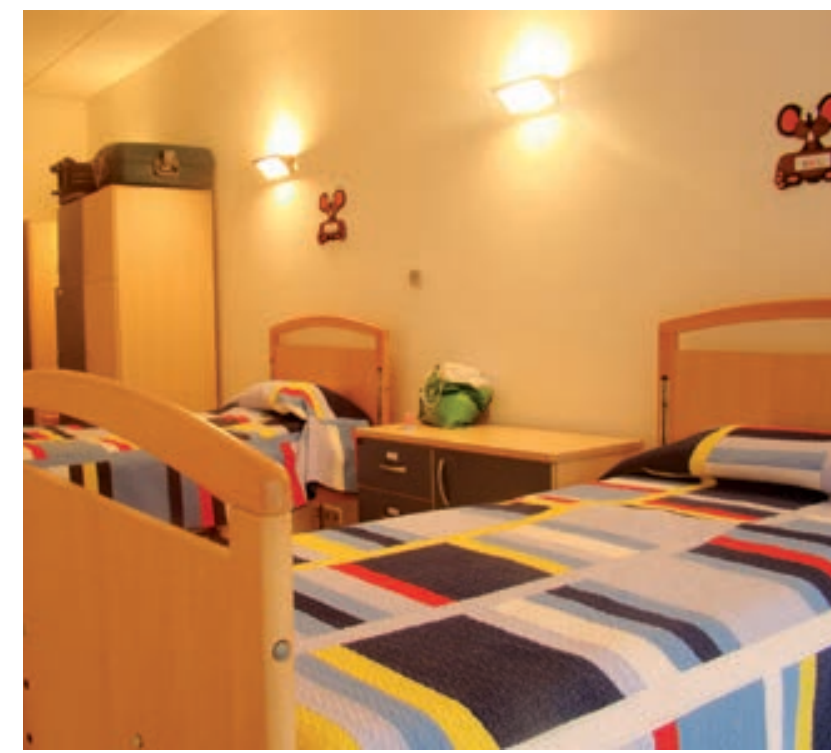

- Se informa sobre convocatorias que pueden ser de interés general, como becas, campamentos abiertos, etcétera. Y se colabora en la gestión de las mismas siempre que es necesario.

- Promueven la dinámica participativa de los padres (escuela con familias).

- Orientan a las familias tanto en demandas puntuales como en todos los procesos relacionados con la mayoría de edad del alumno (incapacidad, pensión, centro, etcétera).

- Asesoran y gestionan ayudas económicas destinadas a la adquisición de ayudas técnicas.

- Facilitan y fomentan el ocio externo del alumno.

En relación con los recursos externos:

- Realizan visitas a centros en función del objetivo que se plantea con el alumno (centros de adultos, centros escolares, centros con plazas concertadas con el Instituto Madrileño de la Familia y el Menor (IMFM), etcétera). Las visitas de profesionales de otras entidades son frecuentes en este centro $y$ son las PTSC las que prestan esta atención.

- La coordinación con entidades privadas y públicas es una constante en su actividad laboral, tanto con servicios vinculados a la educación como a otros sistemas de bienestar. Es una herramienta fundamental para lograr objetivos en su intervención. En este apartado hay que mencionar algunos de los más relevantes, ya que es muy extensa esta red social:

a. Con los profesionales de servicios sociales, que son una pieza básica e imprescindible. Algunas unidades familiares cuando sus hijos se incorporan al centro ya cuentan con intervención previa de estos dispositivos y en otras se propone desde el departamento de trabajo social, cuando se valora necesario. Al ser de ámbito provincial la relación profesional que se establece es muy 


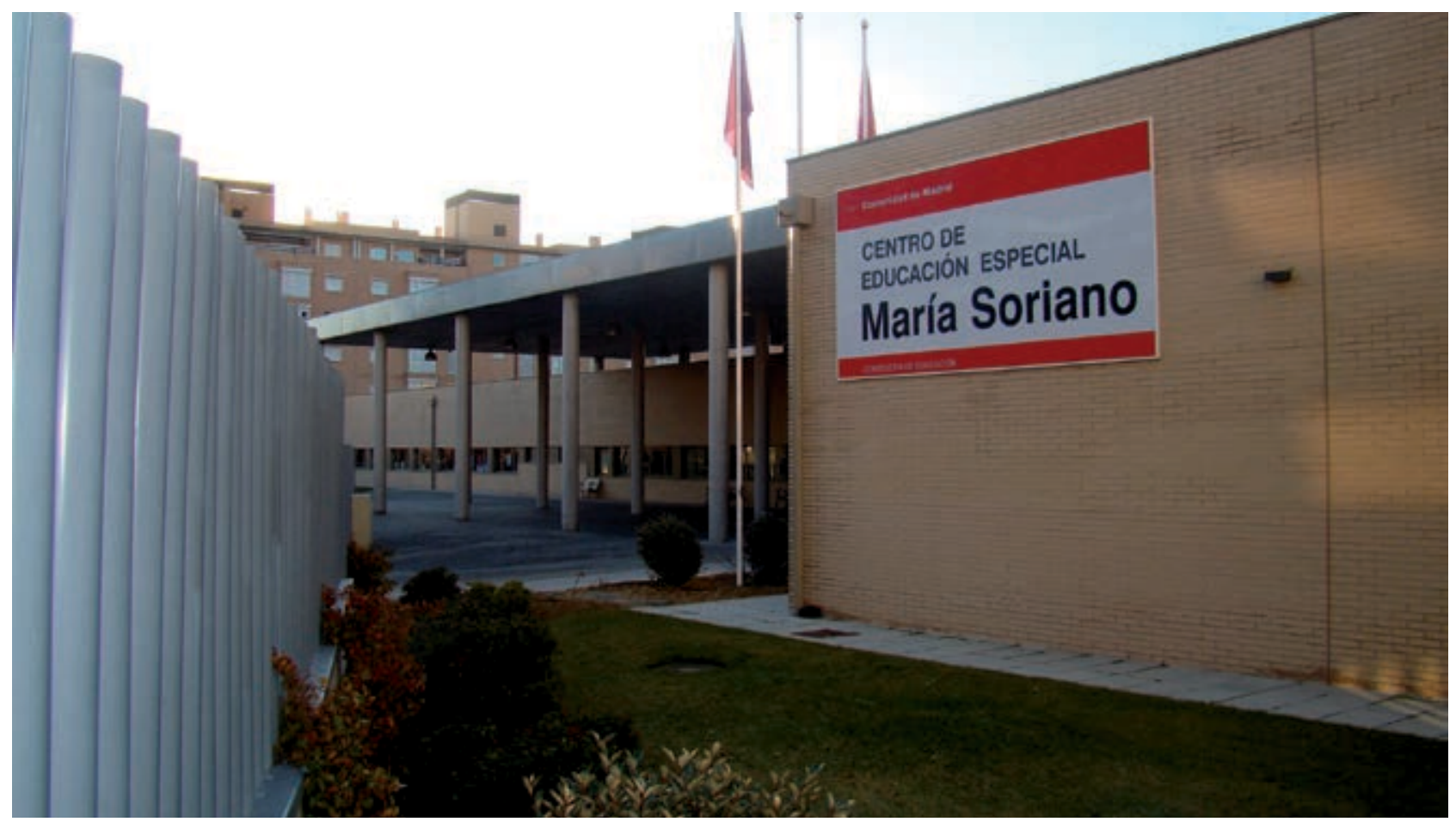

amplia, ya que el alumnado existente es de diferentes distritos de Madrid y de otros municipios de la Comunidad de Madrid.

b. Con profesionales del IMFM de la Comunidad de Madrid. Fundamentalmente con técnicos y con la responsable del área de coordinación de centros. Un grupo de alumnos significativo cuentan con una medida de protección, tutela o guarda de la Comunidad de Madrid.

c. Con los profesionales de sistema de salud. Actualmente existe un convenio entre la Unidad de Cuidados Paliativos del Hospital Niño Jesús y nuestro centro. También se trabaja estrechamente con las trabajadoras sociales de los principales hospitales de Madrid.

d. Con la cooperativa redes a través del programa "Caixa-Proinfancia” de la Fundación La Caixa. Un número destacado de alumnos y de familias han sido beneficiarios de este programa que además ha favorecido la consecución de objetivos relacionados con dinámicas socio familiares.

e. Con la Fundación Prójimo Próximo y la Fundación sobre Ruedas.

f. Con profesiones del ámbito educativo que son los que intervienen en la valoración, determinación de la modalidad educativa del alumno y proponen su escolarización en el centro: los equipos de atención temprana, los equipos de orientación educativa y psicopedagógica y los departamentos de orientación de otros colegios.

Como cierre de esta aproximación a nuestra labor profesional y reconociendo la dificultad de sintetizar el contenido de este documento, nos gustaría destacar el impacto que la discapacidad tiene sobre las familias, los esfuerzos que tienen que realizar los familiares y cuidadores de nuestros alumnos (grandes dependientes), que en muchos casos sufren serias dificultades sociales añadidas (económicas, familiares, laborales, de salud, etcétera). Estas familias son la razón de ser de un departamento como el nuestro, brindarles todo el apoyo posible y acompañarles a lo largo de la vida escolar de sus hijos e hijas, es nuestra principal función.

\section{Para saber más}

- Red de Centros de Educación Especial: http://masjuntosmasespeciales.blogspot.com.es/

- Palacios Sánchez, J. (coord.) (1990). Historia del Colegio Público de Educación Especial Reeducación de Inválidos. Antiguo INRI. Madrid: MEC-ONCE.

- Pujolas Maset. P. (2008). Nueve ideas clave: el aprendizaje cooperativo. Barcelona: Grao.

- Torres González, J. A. (2004). Educación Especial: centros educativos y profesores ante la diversidad. Pirámide.

- VV.AA. (2007). Manual de Tutoría y Orientación en la Diversidad. Madrid: Pirámide.

\section{hemos hablado de:}

Educación especial; discapacidad motórica; trabajo social; trabajo interdisciplinar; intervención social.

Este artículo fue solicitado por PADRES Y MAESTROS en enero de 2014, revisado y aceptado en abril de 2014 para su publicación. 